\title{
Pulmonary and retroperitoneal benign metastasizing leiomyoma
}

\author{
Su Yeon Lim, Joon Cheol Park, Jin Gon Bae, Jong In Kim, Jeong Ho Rhee \\ Department of Obstetrics and Gynecology, Keimyung University School of Medicine, Daegu, Korea
}

Benign metastasizing leiomyoma (BML) is a rare disease, which usually occurs in women with a history of a prior hysterectomy or myomectomy for benign uterine leiomyoma, and has the potential to metastasize to distant sites, such as the lung, lymph nodes, muscular tissue, heart, or retroperitoneum. These lesions are slow-growing, asymptomatic, and usually found incidentally. The prognosis of BML is also excellent. However, there has been debate on the origin and the correct classification of BML, and there are no guidelines for the treatment of BML. We report here on a rare case of BML in both the retroperitoneal cavity and lung in a 48-year-old woman with a history of hysterectomy due to histologically benign uterine leiomyoma. The patient underwent retroperitoneal mass excision and bilateral salpingo-oophorectomy, and then wedge biopsy of two pulmonary nodules was performed additionally 9 days later. Until now, there has been no sign of recurrence and the patient remains asymptomatic. To our knowledge, pulmonary BML is rare and the co-existence of the retroperitoneal metastases after previous hysterectomy is even rarer.

Keywords: Leiomyoma; Lung; Metastasis; Retroperitoneal Mass; Human

\section{Introduction}

Uterine leiomyoma is the most common gynecological tumor, and it usually occurs in the fourth and fifth decades of life. It usually grows during a women's reproductive period and frequently regresses after menopause. However, uterine smooth-muscle tumors with unusual growth patterns, such as benign metastasizing leiomyoma (BML), intravenous leiomyomatosis and leiomyomatosis peritonealis disseminata, rarely exist. Of them, BML has been described as originating from the metastasis of the histologically benign uterine leiomyoma and is an uncommon lesion characterized by the presence of multiple smooth-muscle nodules frequently located in the lung, lymph nodes, or abdomen [1, 2]. Depending on their location, different terminologies, including intravenous leiomyomatosis (when found in

Received: Jun 7, 2011 - Revised: Aug 23, 2011 • Accepted: Sep 5, 2011 Corresponding author: Jeong Ho Rhee

Department of Obstetrics and Gynecology, Keimyung University School of Medicine, 216 Dalseong-ro, Jung-gu, Daegu 700-712, Korea

Tel: +82-53-250-7871 Fax:+82-53-250-7599 E-mail:r1670416@dsmc.or.kr

This is an Open Access article distributed under the terms of the Creative Commons Attribution Non-Commercial License (http://creativecommons.org/licenses/by-nc/3.0/) which permits unrestricted non-commercial use, distribution, and reproduction in any medium, provided the original work is properly cited. vascular channels) and disseminated peritoneal leiomyomatosis (when found in the peritoneal cavity) have been used to describe leiomyomas found outside the confines of the uterus [1].

We describe a rare case of the retroperitoneal and pulmonary BML occurring simultaneously in a 48-year-old woman with a history of hysterectomy due to histologically benign uterine leiomyoma.

\section{Case report}

A 48-year-old woman, gravida 2, was referred to our outpatient clinic for the evaluation of an incidental pelvic mass and multiple lung nodules. She was asymptomatic and did not suffer from abdominal pain, loss of weight, or any other respiratory symptoms like hemoptysis, purulent sputum or exertional dyspnea. Her past medical history was remarkable in that she had had a myomectomy 20 years earlier and then underwent a total abdominal hysterectomy 8 years later because of recurrent multiple uterine leiomyomas. In the initial physical and pelvic examination, a huge firm mass occupying the pelvic cavity was detected. On vaginal ultrasonography, a solid mass with myoma echotexture mass $(14 \times 15 \mathrm{~cm}$ in diameter) filling the pelvic cavity was identified and both ovaries were of normal ap- 
pearance. Chest radiography revealed that it was well-defined, with several nodules in both lung fields and the chest computed tomography $(\mathrm{CT})$ confirmed the aforementioned findings of uncertain origin (Figure 1). Pelvic CT and magnetic resonance image (MRI) showed a lobulating retroperitoneal mass measuring $148 \times 100 \times 154 \mathrm{~mm}$ at the right posterior aspect of the vaginal stump in continuity with the vaginal apex (Figure 2). Routine laboratory investigations and tumor markers including alpha-fetoprotein, CA 19-9 and CA-125, were revealed to be within normal range. Fine needle aspiration of the lung nodule was performed, but the sample obtained from the fine needle aspiration was inadequate for tissue diagnosis. Therefore, the retroperitoneal mass excision and bilateral salpingo-oophorectomy were performed, followed by wedge biopsy of two pulmonary nodules 9 days later. An intraoperative frozen section from the retroperitoneal tumor suggested a leiomyoma.

There was no necrosis or degeneration on the gross or microscopic examination (Figure 3). The microscopic findings of the lung and retroperitoneal masses showed nodular proliferation of spindle cells (Figure 4). The mitotic index was low (less than 1 per 10 high-power fields) and the proliferation index defined by Ki-67 immunostaining (1:200; DAKO, Glostrup, Denmark) was very low (less than 1\%). The spindle cells showed diffuse immunopositivity for smooth muscle actin (1:200; DAKO, Carpinteria, CA, USA), but neither nuclear atypia nor mitotic activity were identified. The immunohistochemical staining for estrogen and progesterone receptors were positive in the pul- monary nodules and the retroperitoneal mass. On the basis of these findings, we diagnosed the pulmonary nodules and the retroperitoneal lesion as BML. Until now, there has been no sign of recurrence and the patient remains asymptomatic.

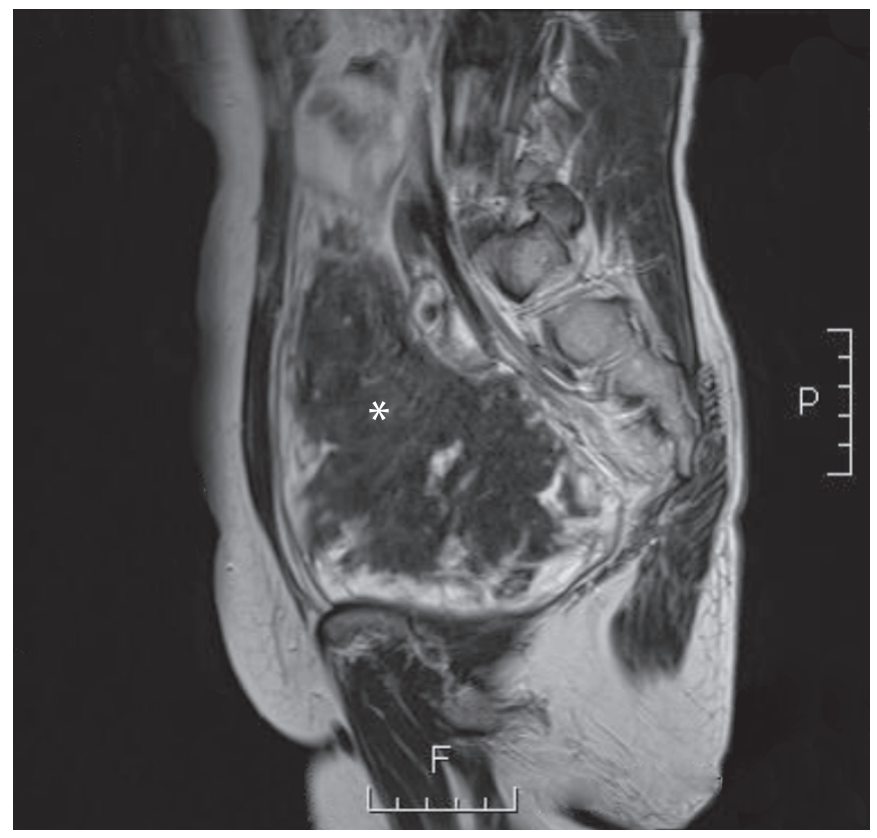

Figure 2. Magnetic resonance image showed a lobulating retroperitoneal mass $\left(^{*}\right)$ in the pelvic cavity.
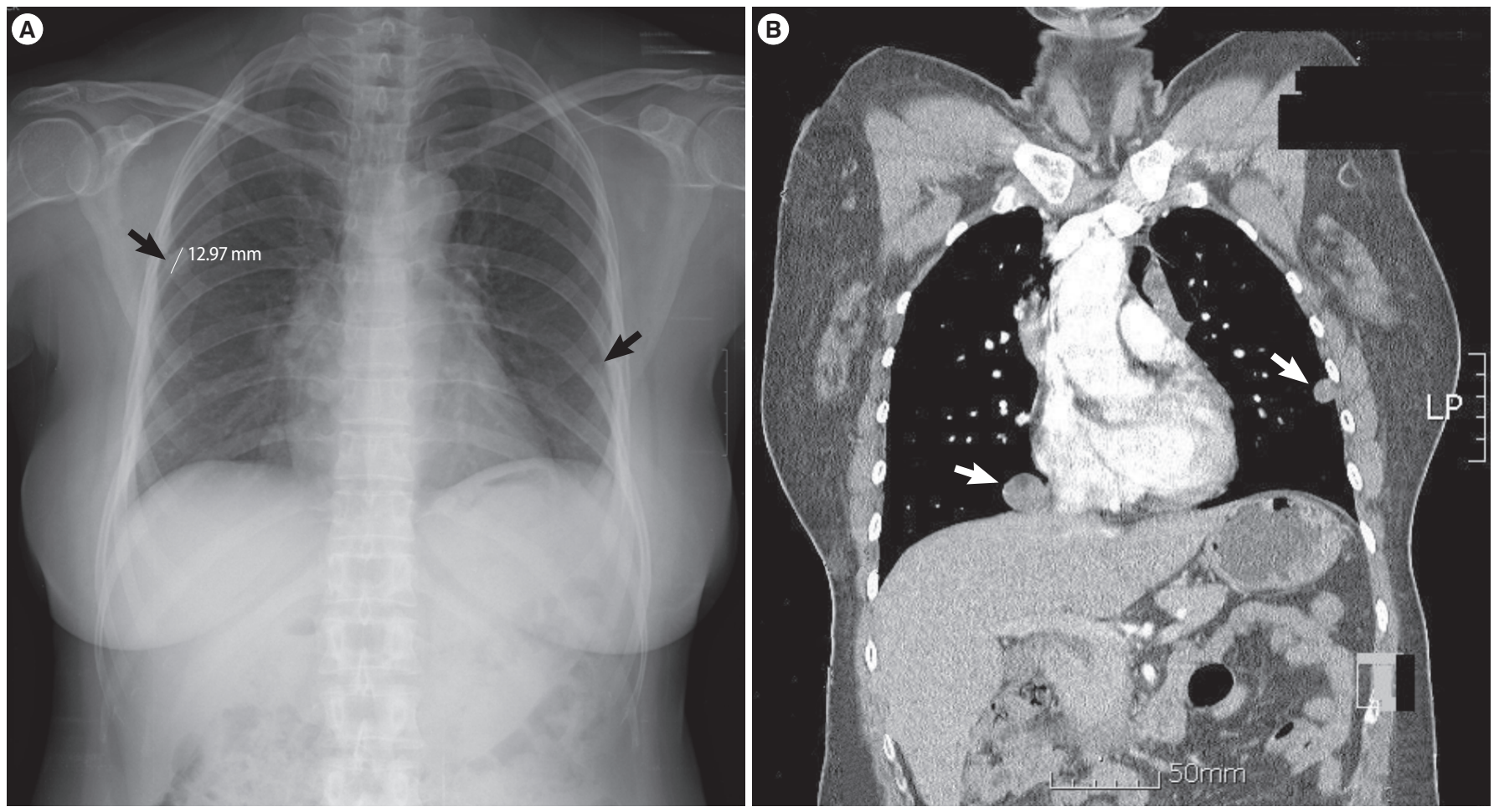

Figure 1. A chest radiograph $(A)$ and computed tomography scan (B) revealed multiple peripheral nodules in the both lungs. 

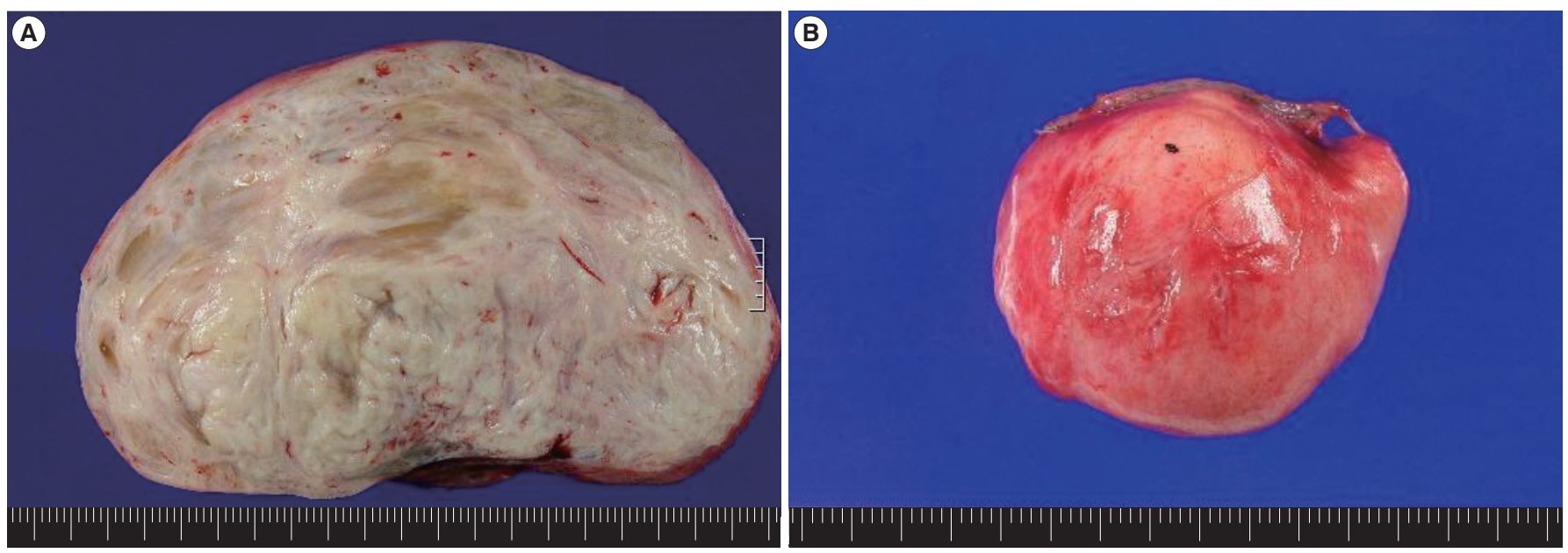

Figure 3. Gross findings of the retroperitoneal mass (A) and pulmonary nodule (B).
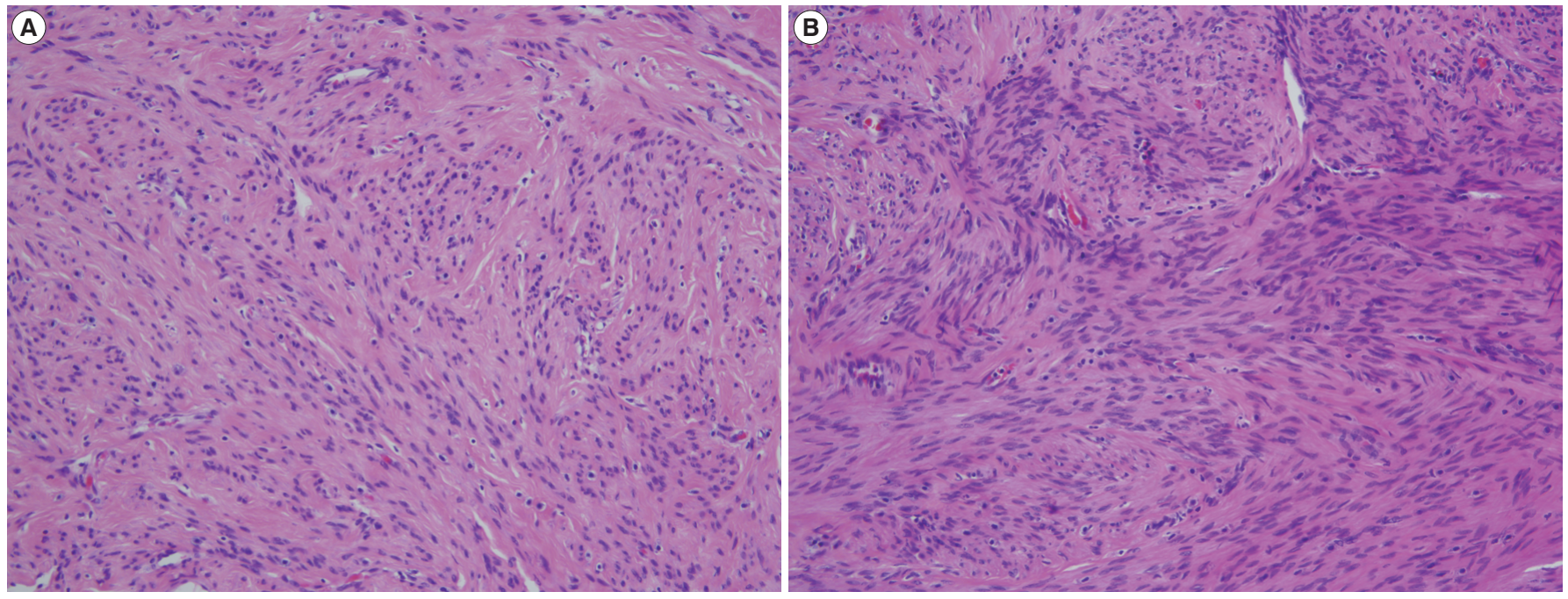

Figure 4. Microscopic findings of the retroperitoneal mass (A) and pulmonary nodule (B). The lesions contain spindle-shaped smooth muscle cells (H\&E stain, $\times 200)$.

\section{Discussion}

Benign metastasizing leiomyoma is rare disease, which usually occurs in women with a history of a prior hysterectomy or myomectomy for benign uterine leiomyoma, and has the potential to metastasize to distant sites, such as the lung, skin, bone, mediastinum, lymph nodes, muscular tissue, heart, and retroperitoneum [3]. These lesions are slow-growing, asymptomatic, and usually found incidentally. In one study, the mean duration between the hysterectomy and the appearance of the lung lesions was 15 years (range, 3 months to 20 years) [4]. To our knowledge, our case is the first report of metastatic lesion in the retroperitoneal cavity and lung occurring simultaneously in Korea, although 5 other cases of metastases to the lung alone after hysterectomy have been reported in Korea [5-9]. The term "BML" was used based on the presupposition that these tumors originate from the metastasis of the histologically benign-appearing uterine leiomyoma that cannot, based upon morphological criteria alone, be recognized as its malignant counterpart, leiomyosarcoma [1]. Actually, it may represent a heterogenous group of smooth muscle tumors, ranging from leiomyoma to low grade leiomyosarcoma [10]. However, there has been debate on the origin and the correct classification of BML, and several hypotheses for the pathogenesis of BML have been proposed. Some investigators have assumed that BML is a benign uterine lesion that is spread via a hematogenous route to the lungs or other organs, in that a majority of patients have undergone myomectomy or hysterectomy suggesting surgically induced hematogenous spread [11]. Supporting this theory is the hormone dependence of both the uterine and the pulmonary lesion. However, the lesion in the lung might take up to 20 years to grow large enough to be detectable. Others groups have argued that uterine lesions and 
the associated BML are clonal proliferations, as it is generally recognized as one mechanism by which endometriosis can be found at distant sites [11, 12]. Patton et al. [11] reported that pulmonary and uterine leiomyomas showed identical patterns of androgen receptor allelic inactivation, indicating that they were monoclonal in origin. Also, they showed that the telomere length measured by fluorescence in situ hybridization in both tumors was long or very long indicating that significant telomere shortening is not a crucial step for developing metastases in these tumors. Therefore, this may be a better theory for describing the truly benign process of BML. Other authors have claimed that all BMLs represent misdiagnosed low grade leiomyosarcomas. Paley and Fornasier [13] reviewed 8 cases of leiomyosarcoma metastasizing to the bone; the primary lesion of the uterus was first diagnosed as leiomyoma but after review appeared to be an underdiagnosed low grade leiomyosarcoma. Although there are limitations in histopathologic testing, they suggested that a definite diagnosis of BML should only be made after careful review of the numerous samples of the primary uterine leiomyoma to exclude small foci of missing sarcoma [14].

Currently there are no guidelines for the treatment of BML due to the limited number of reported cases. Treatment methods include surgical resection of the BML lesion and careful observation. Also, removal of estrogen stimulation by bilateral oophorectomy or hormonal therapy using a gonadotropin-releasing hormone agonist, progesterone, selective estrogen receptor modulator, or aromatase inhibitor has been suggested as the best option for unresectable metastatic lesions [3]. The rationale for the use of hormonal therapy is based on the presence of estrogen and progesterone receptors in both primary tumors as well as in the metastatic nodules [10]. However, because BML could recur after menopause and despite previous oophorectomy, such patients should have prolonged surveillance after surgical removal or hormonal suppression [1]. In addition, tumor resection and biopsy is essential to establish a diagnosis of BML because malignant tumors may exhibit a similar appearance at imaging or clinical examination [2].

To our knowledge, our case is the first report of BML with a huge mass in the retroperitoneal cavity and multiple pulmonary nodules occurring simultaneously in Korea. Although pulmonary BML is rare and the co-existence of the retroperitoneal metastases after previous hysterectomy is even rarer, we suggest that such patients should have prolonged surveillance and careful follow-up.

\section{Conflict of interest}

No potential conflict of interest relevant to this article was reported.

\section{References}

1. Awonuga AO, Rotas M, Imudia AN, Choi C, Khulpateea N. Recurrent benign metastasizing leiomyoma after hysterectomy and bilateral salpingo-oophorectomy. Arch Gynecol Obstet 2008; 278:373-6.

2. Vaquero ME, Magrina JF, Leslie KO. Uterine smooth-muscle tumors with unusual growth patterns. J Minim Invasive Gynecol 2009;16:263-8.

3. Moon H, Park SJ, Lee HB, Kim SR, Choe YH, Chung MJ, et al. Pulmonary benign metastasizing leiomyoma in a postmenopausal woman. Am J Med Sci 2009;338:72-4.

4. Kayser K, Zink S, Schneider T, Dienemann H, Andre S, Kaltner H, et al. Benign metastasizing leiomyoma of the uterus: documentation of clinical, immunohistochemical and lectin-histochemical data of ten cases. Virchows Arch 2000;437:284-92.

5. Park CB, Suh JH, Chang YH, Moon SW, Cho KH, Wang YP, et al. Benign metastasizing leiomyoma of lung: a case report. Korean J Thorac Cardiovasc Surg 2001;34:422-5.

6. Lee EJ, Jeong HC, Lee SY, Kim JH, Lee SY, Shin C, et al. 2 cases of a benign pulmonary metastasizing leiomyoma. Tuberc Respir Dis 2009;67:551-5.

7. Kwon YI, Kim TH, Sohn JW, Yoon HJ, Shin DH, Park SS. Benign pulmonary metastasizing leiomvomatosis: case report and a review of the literature. Korean J Intern Med 2006;21:173-7.

8. Hwang JK, Park KY, Park JW, Park JK, Jeong SH, Jeong JB, et al. A case of benign metastasizing leiomyoma in the lung. Tuberc Respir Dis 2000;49:231-6.

9. Kang SA, Choi SI, Kim YA, Kim CJ, Yang DG, Kang JH, Kie JH, et al. A case of benign metastasizing pulmonary leiomyoma. Tuberc Respir Dis 2005;58:614-8.

10. Goyle KK, Moore DF Jr, Garrett C, Goyle V. Benign metastasizing leiomyomatosis: case report and review. Am J Clin Oncol 2003; 26:473-6.

11. Patton KT, Cheng L, Papavero V, Blum MG, Yeldandi AV, Adley BP, et al. Benign metastasizing leiomyoma: clonality, telomere length and clinicopathologic analysis. Mod Pathol 2006;19:130-40.

12. Nuovo GJ, Schmittgen TD. Benign metastasizing leiomyoma of the lung: clinicopathologic, immunohistochemical, and microRNA analyses. Diagn Mol Pathol 2008;17:145-50.

13. Paley D, Fornasier VL. Leiomyomatosis metastasizing to the spine. J Bone Joint Surg Am 1984;66:630.

14. Rakhshani N, Hormazdi M, Abolhasani M, Shahzadi M. Benign metastasizing leiomyoma of the uterus. Arch Iran Med 2007;10: 97-9. 